\title{
UNA APROXIMACIÓN A LAS VARIABLES PREDICTORAS DEL RENDIMIENTO ACADÉMICO EN ESTUDIANTES DE SECUNDARIA
}

\author{
PREDICTIVE VARIABLES OF ACADEMIC ACHIEVEMENT \\ IN SECONDARY EDUCATION STUDENTS
}

\author{
Ramón González*, Paula Mendiri** y Alicia Arias*** \\ Universidade da Coruña
}

\begin{abstract}
RESUMEN
En este artículo presentamos el proceso y los resultados de un estudio de investigación sobre una serie de variables cognitivo-motivacionales en una muestra de estudiantes de Educación Secundaria. Mediante diferentes análisis de regresión lineal múltiple obtenemos las variables predictoras del rendimiento académico, analizando y discutiendo los resultados obtenidos.
\end{abstract}

Palabras clave: autoconcepto académico, metas académicas, estrategias de aprendizaje y rendimiento académico.

\section{ABSTRACT}

This work presents the process and results of an empirical research several carried out on cognitive and motivational variables in a sample of secondary education students. Through different multiple linear regression analyses we obtain the predictive variables of academic achievement, analysing and discussing the outcomes.

Key words: academic self-concept, academic goals, learning strategies and academic achievement.

* Ramón González Cabanach. Catedrático y Profesor del Departamento de Psicología Evolutiva y de la Educación de la Facultad de Ciencias de la Educación de la Universidade da Coruña.

** Paula Mendiri. Profesora del Departamento de Filosofía y Métodos de Investigación de la Facultad de Ciencias de la Educación de la Universidade da Coruña.

*** Alicia Arias. Profesora del Departamento de Filosofía y Métodos de Investigación de la Facultad de Ciencias de la Educación de la Universidade da Coruña. 


\section{Introducción}

Numerosos estudios (p. e., González y Tourón, 1992; González Cabanach, 1994; Núñez et al., 1998; Valle, 1997) han demostrado que son varios los factores y variables que influyen en el rendimiento académico, desde características individuales como la inteligencia, estilos cognitivos, la personalidad del estudiante, las orientaciones motivacionales, el autoconcepto, las atribuciones causales y los enfoques de aprendizaje hasta el contexto en el que se lleva a cabo ese aprendizaje. El autoconcepto es un constructo psicológico que se refiere a las percepciones que una persona mantiene respecto de sí misma (Shavelson, Hubner y Stanton, 1976). En cuanto a su conceptualización, varios autores (p. e., Hattie, 1992; Marsh, 1987) se apoyan en el modelo multidimensional y jerárquico de Shavelson, Hubner y Stanton (1976) que distingue los componentes académicos y no académicos del autoconcepto y que posteriormente sería revisado por otros autores (p. e., Byrne y Shavelson, 1986).

Las atribuciones causales se refieren a las percepciones sobre por qué ocurren los acontecimientos. La teoría de la atribución fue introducida por Heider (1958) para explicar las atribuciones causales que realizaban las personas de los acontecimientos sociales. Weiner $(1979,1986)$ propuso una teoría de la atribución de la motivación que fue el punto de partida de muchas investigaciones en el contexto de los resultados académicos, que se proponían comprobar cómo los estudiantes diferían en su modo de explicar las causas de sus éxitos y fracasos académicos. Se considera que el proceso atribucional tiene una influencia significativa en el comportamiento cognitivo, afectivo y en el rendimiento de los estudiantes (Weiner, 1986). Según Weiner (1979), las cuatro causas a las que, mayoritariamente, se atribuyen los resultados académicos son la capacidad, el esfuerzo, la dificultad de la tarea y la suerte. Otros estudios encontraron que las causas del rendimiento eran el interés, la estado de ánimo, la ayuda de los otros y el apoyo familiar (Elig y Frieze, 1975). Estas causas percibidas pueden ser clasificadas en tres dimensiones, como son lugar de causalidad, estabilidad y controlabilidad (Weiner, 1986). La capacidad y el esfuerzo están comúnmente clasificadas por los investigadores como atribuciones internas, mientras que la dificultad de la tarea y la suerte se consideran atribuciones externas. Mientras que algunas investigaciones han demostrado que las atribuciones están asociadas con el rendimiento académico (Findley y Cooper, 1983), no está muy clara la naturaleza causal de esta relación. Marsh (1984) mantenía que el rendimiento influía en las atribuciones, y Covington y Omelich (1984) argumentaban lo contrario.

Según Nicholls (1989), los estudiantes pueden presentar diferentes orientaciones de aprendizaje, lo que conlleva la obtención de satisfacciones en situaciones de aprendizaje a través de diferentes vías: incrementando las propias competencias haciendo progresos en la realización de las tareas (orientación a la tarea), rindiendo mejor o, por lo menos, no peor que los otros (orientación al «yo»), o haciendo lo menos posible (evitación de la tarea). La clasificación de las metas en las que nos hemos centrado es la ofrecida por Dweck (1986) y Dweck y Leggett (1988) que considera, por un lado, las metas de aprendizaje y, por otro, las metas de rendimiento. Los sujetos orientados a las primeras se interesan por incrementar su competencia y mejorar su proceso de aprendizaje y los orientados a las segundas buscan la obtención de juicios favorables sobre su competencia. Además, estudios posteriores diferencian en este segundo tipo de metas, las de refuerzo social y las de logro.

Numerosos estudios han demostrado que elevados niveles de orientación a la tarea están asociados con estrategias de estudio más efectivas y con orientaciones más positivas para 
continuar trabajando. Mientras que Nicholls presentó estas orientaciones en términos de rasgos relativamente estables, también se ha demostrado que la orientación motivacional, o más propiamente, los tipos de metas pueden estar influidos pos la práctica de clase (Rogers, 1995). Nicholls (1984) destacó la posibilidad de que los sujetos diferenciaran entre capacidad y esfuerzo, mientras que Dweck (1986) destacó la distinción entre la capacidad como estable o modificable. La concepción de la capacidad como fija y estable está asociada con una motivación menos adaptativa.

En definitiva, consideramos que el estudiante tiene un papel activo en todo el proceso de aprendizaje y que las diferencias que pueden surgir en el rendimiento académico no pueden ser atribuidas, únicamente, a las habilidades sino que son debidas a la interacción que se establece entre variables cognitivas, afectivas y motivacionales.

\section{Objetivos}

A partir de estas consideraciones, con este estudio exploratorio, nos proponemos identificar las variables cognitivo-motivacionales más relevantes que influyen en el rendimiento académico de los alumnos de Educación Secundaria, así como la relación existente entre ellas. Para ello nos guiaremos por una serie de objetivos específicos que formulamos de la siguiente manera: $1^{\circ}$. conocer qué variables motivacionales predicen mejor el autoconcepto académico de los estudiantes; $2^{\circ}$. identificar los tipos de metas de estudio que guían el aprendizaje de estos alumnos; $3^{\circ}$. conocer qué variables predicen la orientación de los estudiantes a los diferentes tipos de metas; $4^{\circ}$. conocer las variables que mejor predicen la utilización de estrategias positivas de aprendizaje; $5^{\circ}$. conocer las variables que mejor predicen el rendimiento académico.

\section{Muestra}

La muestra está formada por 338 sujetos de diferentes centros de A Coruña, que fueron seleccionados mediante muestreo probabilístico aleatorio por conglomerados en el que la unidad muestral es el grupo de clase que ya está formado en el centro educativo. De la muestra total, 148 (43.8\%) son hombres y 190 (56.2\%) son mujeres. Las edades de los encuestados van desde los 13 hasta los 19 años con una media de 15.62 años. Los estudiantes pertenecen a Centros situados en la periferia de A Coruña y en el centro de la ciudad. La mayoría son de carácter público, puesto que nos resultó difícil acceder a los de carácter privado.

\section{Instrumentos}

Pasamos los cuestionarios de autoconcepto SDQII (Marsh, H. W., Parker, J. y Barnes, J., 1985), de motivación de logro (Hayamizu y Weiner, 1991), el inventario de habilidades de aprendizaje de estudio, LASSI (Weinstein, 1987) y la escala de evaluación de procesos y contextos motivacionales (Núñez y González-Pienda, 1994). 


\section{Procedimiento}

Se trata de un estudio transversal, no experimental, descriptivo y correlacional. En función del método de recogida de información se trata de un diseño de encuesta (Arnau, 1995), mediante la técnica del cuestionario. En cuanto a la recogida de la información, el primer paso consistió en la puesta en contacto con los Directores de los Centros de Educación Secundaria, seleccionados al azar, para explicar los principales objetivos del trabajo y discutir sobre la mejor manera de que los alumnos no perdieran un gran número de horas de clase, ya que cada uno debía de contestar varios cuestionarios y eso requería la pérdida de horas lectivas. Además de la reticencia de los Directores, por solicitar a los profesores sus horas de clase, nos encontramos con el temor, por parte del profesorado, de que pudieran ser objeto de evaluación y, aun viendo los cuestionarios y comprobando que ninguna de las preguntas iba dirigida hacia este sector, se percibía mucha desconfianza y recelo. Esto hizo que la muestra fuera más delimitada de lo planificado en un primer momento, lo que nos llevó a interpretar los datos con cierta cautela y quedarnos en un estudio exploratorio que nos sirviera de base para otros posteriores. La aplicación de los instrumentos de recogida de datos fue llevada a cabo por la misma persona, que explicó muy brevemente a los estudiantes los objetivos del trabajo y se ocupó de que se contestaran a todas las preguntas planteadas en los cuestionarios y de aclarar las dudas que les surgían. Se realizó de forma colectiva y en horas de clase para evitar que los estudiantes no los contestaran. Las limitaciones con las que cuenta este tipo de instrumento se ciñen a problemas de validez, debido a una posible falta de sinceridad en las respuestas de los encuestados y a una tendencia a ajustarse a patrones de deseabilidad social. Por eso se recalcó en varias ocasiones la importancia de ser sinceros en las respuestas y se insistió en que no había respuestas «correctas» o «incorrectas». Una vez recogidos los cuestionarios, se revisaron para eliminar los que no estaban correctamente contestados o incompletos, de manera que de la muestra invitada, formada por 380 alumnos, pasamos a obtener una muestra real de 338.

\section{Resultados}

\section{Variables predictoras del autoconcepto académico}

Considerado el autoconcepto como pieza central del proceso motivacional, vamos a comprobar qué variables cognitivo-motivacionales predicen mejor el autoconcepto académico general de los estudiantes mediante un análisis de regresión lineal múltiple. Las variables independientes que vamos a introducir en el modelo son el autoconcepto matemático, el autoconcepto verbal, que son considerados como los factores más significativos en el autoconcepto académico del estudiante, el autoconcepto en la relación con los padres, que es una de las fuentes importantes dentro del grupo de la valoración de los otros significativos en la formación del autoconcepto, la capacidad percibida o creencia de que se dispone de las capacidades necesarias para realizar con éxito las conductas requeridas para producir los resultados deseados, el rendimiento o logros previos y la atribución de los resultados académicos al esfuerzo (causa interna e inestable).

Según los resultados obtenidos, a los estudiantes que tienen una buena imagen en cuanto a sus habilidades en matemáticas, en el área del lenguaje, que presentan una buena ima- 
gen de la interacción con sus padres, que creen que la causa de su rendimiento académico es el esfuerzo que dispensan a la hora de realizar las tareas, con buenos resultados académicos en los cursos previos y, por último, que consideran que tienen una buena capacidad para el trabajo escolar, se les puede predecir un mejor autoconcepto académico que a los estudiantes que no presentan esas características. Por lo tanto, la variable que mejor predice es autoconcepto matemático y la que menos, la capacidad percibida (Figura 1). La medida del grado de asociación entre el autoconcepto académico general y el conjunto de variables independientes es $\mathrm{R}=665$. El coeficiente de determinación tiene un valor de .442 , por lo que el modelo explica un $44,2 \%$ de la variabilidad del criterio «autoconcepto académico general».

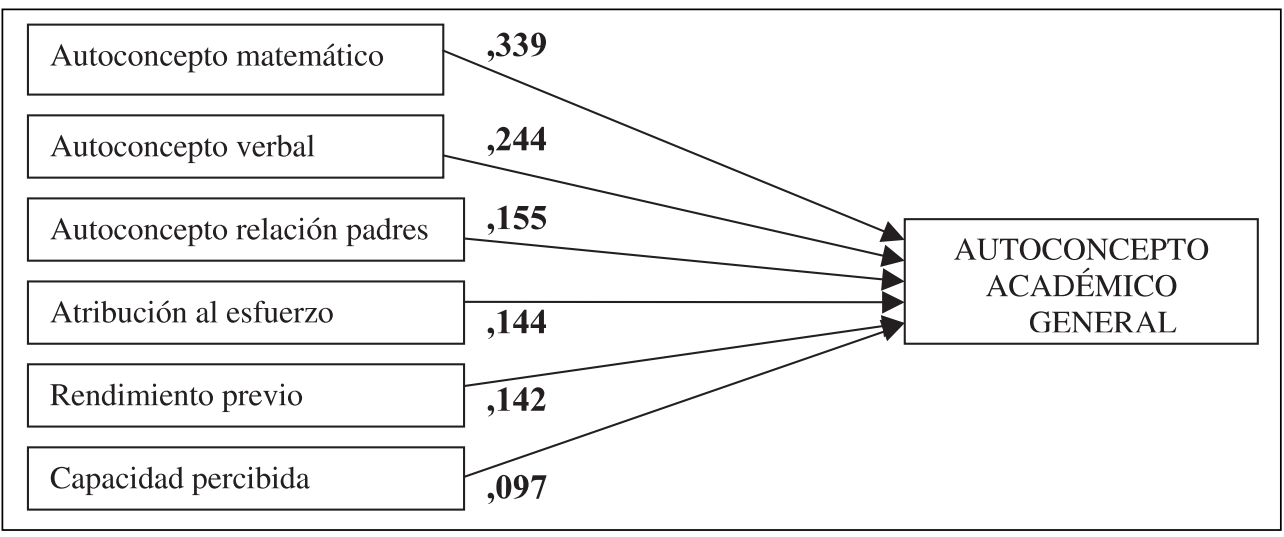

FIGURA 1.

Coeficientes de correlación estandarizados de cada una de las variables independientes.

\section{Orientación de los estudiantes a las metas académicas y variables predictoras de las mismas}

Vamos a centrarnos en los tipos de metas académicas a los que pueden orientarse los estudiantes que, según Hayamizu y Weiner (1991), se diferencian en metas de aprendizaje (metas intrínsecas) y metas de ejecución o rendimiento (metas extrínsecas), que comprenden las metas de refuerzo social y las metas de logro. Primero observamos a qué tipo de metas tienden más los estudiantes y encontramos que lo hacen, sobre todo, hacia las metas de logro $(M=4,20 ; \mathrm{DT}=0,86)$, es decir, tienden a aprender para obtener buenos resultados en los exámenes y avanzar en los estudios, aunque también presentan una motivación intrínseca expresada en las metas de aprendizaje $(\mathrm{M}=3,18$; DT $=0,86)$ y orientada a incrementar las propias competencias; esto nos vuelve a demostrar que son varias las orientaciones de meta que dirigen al aprendizaje de los estudiantes en este nivel educativo.

Es probable que esta tendencia a estos dos tipos de metas se deba, al menos parcialmente, a la doble caracterización de esta etapa educativa que tiene, por un lado, un carácter terminal de preparación para la vida activa y, por otro, un carácter propedéutico para acceder a otros estudios. Esto puede justificar que los estudiantes estén interesados, no sólo por 
aprender para incrementar sus conocimientos y competencias, sino también en obtener calificaciones elevadas en las diferentes asignaturas, de cara a tener la posibilidad de poder escoger la carrera que les guste e interese estudiar.

En un primer análisis, nos vamos a centrar en las metas de logro y vamos a intentar establecer, con las variables que tenemos y que la pueden predecir, un modelo de regresión lineal. Incluimos, como variables independientes, aquéllas que se refieren a características personales o individuales que pueden influir en la elección de las metas, como son la atribución del rendimiento al esfuerzo (atribución interna), la atribución del rendimiento a la suerte (atribución externa), el autoconcepto académico general, la capacidad percibida y la concepción incremental de la inteligencia. El modelo resultante (figura 2) explica tan sólo un $14,1 \%$ de la variabilidad, por lo que se tendrían que incluir, en posteriores ocasiones, otras variables que contribuyeran a su explicación, por ejemplo, el tipo de objetivos que se establecen en clase, las estrategias de enseñanza del profesor, el ambiente evaluativo o participativo, el estilo de enseñanza, etc. La correlación múltiple entre la variable criterio y las predictoras es de 0,376 .

\begin{tabular}{|l|l|l|}
\hline Autoconcepto académico & $\mathbf{2 3 4}$ & \\
\hline Atribución al esfuerzo & $\mathbf{1 7 6}$ & METAS DE LOGRO \\
\hline $\begin{array}{l}\text { Concepción incremental } \\
\text { de la inteligencia }\end{array}$ & $\mathbf{1 4 0}$ \\
\cline { 2 - 2 } & \\
\hline
\end{tabular}

\section{FIGURA 2. \\ Coeficientes de correlación estandarizados de las variables predictoras con la variable criterio.}

Aunque podríamos prescindir del comentario de estos resultados por su escasa explicación, procederemos a una reflexión crítica, pero sin intención de que se considere como posible conclusión. La variable que mejor predice la orientación a las metas de logro es el autoconcepto académico y la que menos la concepción incremental de la inteligencia. Por lo tanto, según el modelo obtenido, los alumnos que presentan una imagen positiva de sí mismos como estudiantes, que consideran que la causa de sus resultados académicos es el esfuerzo dispensado en la realización de las tareas y que presentan una concepción menos diferenciada de la inteligencia y, por lo tanto, consideran que es modificable mediante el esfuerzo, tenderán a orientarse a las metas de logro. Ahora bien, insistimos en que estas afirmaciones deben ser cautelosas debido a que queda más porcentaje de la variabilidad sin explicar que explicado. Las dos variables eliminadas, dado su escaso poder explicativo, han sido las que se refieren a la atribución externa de los resultados académicos y la capacidad percibida.

\section{Variables predictoras de las estrategias de aprendizaje}

En este punto vamos a centrarnos en otra de las variables que consideramos tiene una notable relevancia en el proceso de aprendizaje: la utilización de estrategias positi- 
vas de aprendizaje, que será la variable criterio de nuestro siguiente modelo. Como variables predictoras, introducimos las metas de aprendizaje, puesto que consideramos que la intención o motivo de comprender el significado de la tarea requiere para su consecución de unas estrategias de aprendizaje que darían lugar al enfoque profundo mediante el que se relacionan las experiencias actuales con los conocimientos previos; las metas de logro, encaminadas a la obtención de elevadas calificaciones, pero unidas a la comprensión y búsqueda estratégica del significado, que daría lugar al enfoque logro-profundo; una serie de características contextuales del proceso de aprendizaje sobre las que va a reflexionar el estudiante a la hora de utilizar unas estrategias de aprendizaje u otras, como son la percepción de los criterios de evaluación, el análisis de las características de la tarea, la percepción del estilo de enseñanza y la percepción del tipo de materia; la atención y concentración, que permiten al estudiante orientar la atención hacia lo que está trabajando; las técnicas de estudio o recursos que se pueden utilizar para facilitar tanto el aprendizaje como el recuerdo del material de estudio, el control y la distribución del tiempo y el uso de estratagemas o la utilización de una serie de recursos para la superación de los exámenes (figura 3). Ahora bien, este modelo explica tan sólo un $31,7 \%$ de la variabilidad del criterio y el coeficiente de correlación múltiple alcanza un valor de 0,563 .

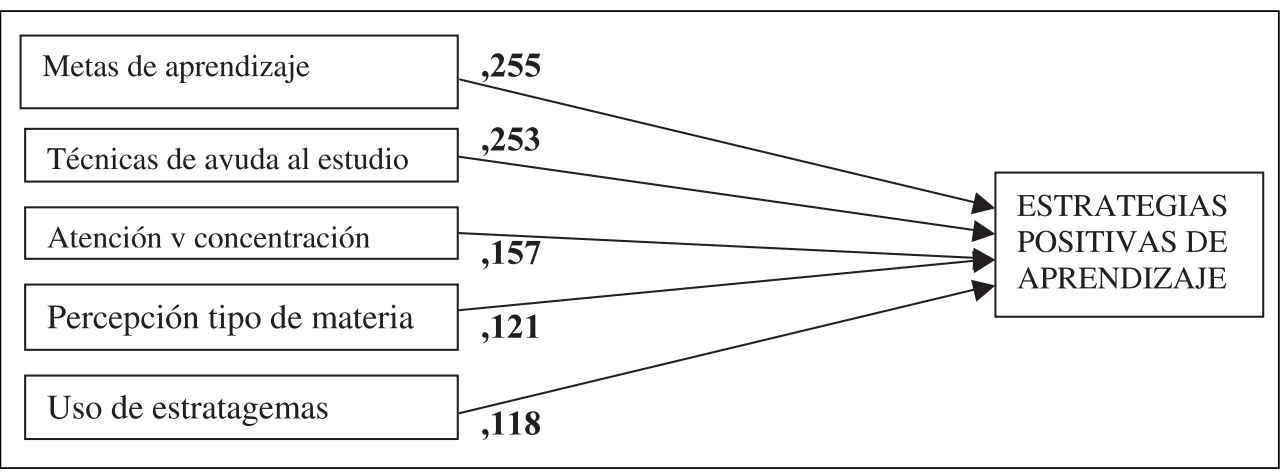

FIGURA 3.

Coeficientes de correlación estandarizados de las variables predictoras con la variable criterio. Modelo general.

\section{Variables predictoras del rendimiento académico}

Por último, vamos a ver las variables que pueden predecir el rendimiento académico entre las siguientes: autoconcepto académico, atribución del rendimiento al esfuerzo y a la capacidad, metas de logro y metas de aprendizaje, rendimiento previo y estrategias positivas de aprendizaje. En el modelo (figura 4), que explica un 57,4\% de la variabilidad del criterio, las variables predictoras resultantes son las anteriormente mencionadas, salvo las metas de aprendizaje y la utilización de estrategias positivas. El coeficiente de correlación múltiple tiene un valor de 0,758 . 


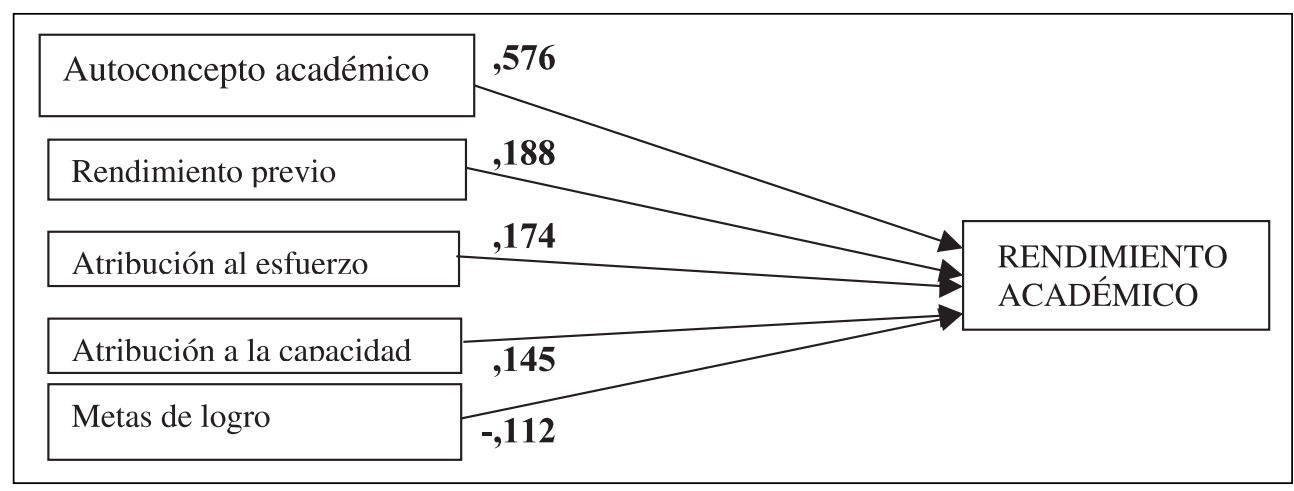

\section{FIGURA 4. \\ Coeficientes de correlación estandarizados de las variables predictoras con la variable criterio. Modelo general.}

\section{Conclusiones}

El primer objetivo hacía referencia al conocimiento de las variables que mejor pueden predecir el autoconcepto académico de los estudiantes y, teniendo en cuenta a todos los sujetos de la muestra, comprobamos que la variable que mejor lo predice es el autoconcepto matemático, y la que menos, la consideración por parte de los estudiantes de que poseen una buena capacidad para el trabajo escolar. Debido a que el modelo explica un $44,2 \%$ de la variabilidad del criterio, habría que introducir otras variables, quizás situacionales. La imagen que tienen de sí mismos como estudiantes 1@s alumn@s, va a depender de la imagen que tienen de su habilidad, implicación e interés en todo lo relacionado con las matemáticas y con el lenguaje. Eso coincide con la afirmación de que los autoconceptos verbal y matemático son considerados como las dimensiones más significativas del autoconcepto académico en la etapa de la preadolescencia (p. e., Marsh y Hattie, 1996). Aunque se puede considerar el interés demostrado por la familia hacia las tareas de los hijos, como un factor positivo en el afianzamiento de una imagen de seguridad como estudiante, va a tener una importancia relativa dependiendo del valor que los estudiantes otorguen a la evaluación de los padres como elementos significativos o no (Brookover, 1967).

Otra variable que predice el autoconcepto académico es la atribución de los resultados académicos al esfuerzo, lo cual puede significar una tendencia optimista o autoprotectora, es decir, ayuda a los sujetos a ofrecer una imagen positiva de sí mismos de manera que, ante los éxitos el autoconcepto, siga mejorando y, ante los fracasos, tengan la posibilidad de pensar en esforzarse más (aunque, posteriormente, no lo hagan), pudiendo emerger sentimientos de esperanza. Las expectativas de autoeficacia o confianza personal de que se pueden ejecutar con éxito las conductas requeridas para producir los resultados deseados, resultan también, aunque en menor medida, predictoras del autoconcepto académico. En este punto queremos aclarar que, mientras que la autoeficacia se refiere a la percepción de las capacidades específicas, el autoconcepto académico es la percepción como estudiante, en general. Además están relacionadas con los resultados académicos, puesto que, si el estudiante obtiene un buen rendimiento en los cursos precedentes, su imagen, como estudiante, 
se verá favorecida; puede fracasar en algún momento, superarlo y mejorar su autoconcepto y también puede fracasar, repetidamente, empeorar su autoconcepto y tener lugar la indefensión aprendida (Seligman, 1975).

En cuanto al segundo objetivo, la identificación de los tipos de metas de estudio que guían el aprendizaje de los estudiantes, encontramos que lo hacen, sobre todo, hacia las metas de logro, es decir, tienden a aprender para obtener buenos resultados en los exámenes y avanzar en los estudios, aunque también presentan una motivación intrínseca expresada en las metas de aprendizaje y orientada a incrementar las propias competencias; esto nos vuelve a demostrar que, en este nivel educativo, son varias las orientaciones de meta que dirigen la actividad escolar que actúa como factor determinante del interés y esfuerzo que el estudiante va a poner en el aprendizaje.

Por lo que se refiere al tercer objetivo, a partir del que se pretende conocer las variables que mejor predicen la orientación de los estudiantes a los diferentes tipos de metas, podemos afirmar que los modelos encaminados a la explicación de la elección, por parte de los estudiantes, de las metas de logro explican muy poco la variabilidad del criterio, por lo cual, para sucesivas ocasiones, habría que introducir, además de características personales de los estudiantes, una serie de variables ambientales y externas que hicieran referencia, por ejemplo, al tipo de objetivos que se establecen en clase (la necesidad, muchas veces, determina las metas), la estructura de la tarea, los sistemas de recompensa, los comentarios del profesor, etc. Como señala Seifert (1997), los factores contextuales o ambientales provocan reacciones emocionales en los estudiantes que, a su vez, inducirán la orientación de éstos hacia las diversas metas.

El porcentaje de variabilidad explicado para las metas de aprendizaje aumenta un poco, pero todavía es pequeño (36,9\%), lo que seguimos atribuyendo a lo señalado anteriormente. Aparece el autoconcepto académico de los estudiantes, lo cual nos hace aproximarnos más a los autores que defienden que las metas que persigue el estudiante están determinadas por la imagen que tiene de sí mismo como estudiante (p. e., Ames, 1992; Covington, 1985; Dweck, 1986; Nicholls, 1984) que a los que afirman que la motivación está determinada por las dimensiones de las atribuciones causales (p. e., Weiner, 1986). Otra variable que aparece en el modelo es la que se refiere a la concepción incremental o menos diferenciada de la inteligencia y que va unida a la idea de que a mayor esfuerzo invertido, mayor capacidad (Nicholls, 1984). Generalmente, estos sujetos están centrados en la tarea, y se autodirigen mensajes para la superación del fracaso que lo consideran como algo de lo que hay que aprender. Su interés va a estar centrado en el proceso de realización de la tarea, y tengan alto o bajo autoconcepto, van a preferir las tareas de dificultad moderada (Nicholls, 1984). En relación con la capacidad percibida, Smiley y Dweck (1994) comprobaron que los alumnos orientados a las metas de aprendizaje, con alta o baja capacidad autopercibida, adoptaban un patrón motivacional adaptativo de reto, y experimentaban afectos más positivos con las tareas que suponían cierto desafío.

Siguiendo con el cuarto objetivo, mediante el que intentamos conocer las variables que mejor predicen la utilización de estrategias positivas de aprendizaje, vemos que el hecho de perseguir la actualización de los propios intereses, adoptando un motivo intrínseco, junto con las estrategias de aprendizaje, forman el enfoque profundo de aprendizaje; es decir, las estrategias positivas de aprendizaje, entre otras actividades, incluyen la selección y organización de la información, el repaso del material de aprendizaje, la relación del nuevo mate- 
rial con el que ya existe en la memoria, de manera que se pueda hacer más significativo y le permita al alumno incrementar sus propias competencias y ampliar sus conocimientos (objetivo de las metas de aprendizaje). Aunque se considera la estrategia como un mecanismo de nivel superior a la técnica, pueden y deben trabajar conjuntamente para producir el resultado de aprendizaje. De hecho, la utilización de las técnicas va a ayudar a los estudiantes a conseguir una lectura más comprensiva mediante el subrayado, los esquemas, resúmenes, síntesis, búsqueda de la idea principal, diagramas, etc. El inconveniente que encontramos con la medición de las estrategias, mediante este cuestionario, es que sólo se refieren a las cognitivas y tenemos que prescindir de otras tan relevantes como las metacognitivas, las de control y gestión de recursos, y las motivacionales.

Uno de los aspectos esenciales del aprendizaje autorregulado es la autosupervisión del propio pensamiento (Lan, 1996) y del comportamiento académico, es decir, los estudiantes consideran que una estrategia cognitiva está siendo efectiva si les ayuda a progresar hacia la meta y si no requiere demasiado tiempo y esfuerzo. Para ello, es necesario mantener la atención y la concentración en la tarea que se está realizando (Mayer, 1992; Pressley y Ghatala, 1990 y Schunk, 1991), de manera que el estudiante consiga estar inmerso en el tema objeto de estudio y excluya todos los demás estímulos que puedan causar distracción. Así, es una de las variables que predice la utilización de estrategias de aprendizaje significativo.

Esto, a su vez, va unido a la motivación, puesto que, para que un estudiante consiga una atención efectiva, tiene que estar interesado en el tema. Por eso es lógico que otra de las variables predictoras de la utilización de estrategias positivas de aprendizaje se refiera a la percepción o análisis de las características de la tarea que, dependiendo de éstas, planificará la acción de una manera u otra y reflexionará sobre el tipo de estrategias más adecuadas para su resolución. Así, un estudiante puede sentir mayor interés por un tipo u otro de materia y pretender comprenderla profundamente mediante la utilización de estrategias positivas de aprendizaje o, por el contrario, estudiarla de manera superficial.

Sin embargo llama la atención que el uso de estratagemas, que consiste en utilizar una serie de recursos para superar los exámenes mediante el mínimo gasto de esfuerzo y tiempo, tenga relación directa con la utilización de estrategias de aprendizaje que, precisamente, lo que permiten es la adquisición de nuevos conocimientos, su comprensión, almacenamiento adecuado, relación con los conocimientos ya existentes y su utilización en el momento oportuno. La explicación que le podemos dar es que se entienda como una de las actividades que comprendidas en las estrategias de elaboración que consiste en formular preguntas y darles una respuesta, de manera que el estudiante pueda reflexionar, más profundamente, sobre la información y relacionar unos datos con otros.

El quinto objetivo hace referencia al conocimiento de las variables que mejor predicen el rendimiento académico, $\mathrm{y}$, encontramos un modelo que explica un 57,4\% de la variabilidad del criterio y que está compuesto por las variables predictoras: autoconcepto académico o imagen que se tiene de uno mismo como estudiante, con una relación mayor que cualquiera de las variables que le siguen; rendimiento previo o los resultados académicos obtenidos en cursos anteriores; atribución del rendimiento al esfuerzo; atribución del rendimiento a la capacidad; $y$, con relación inversa, la orientación a las metas de logro. Esta relación nos parece curiosa porque, como explicamos en el diseño de la investigación, el rendimiento se obtuvo mediante las calificaciones obtenidas por los estudiantes en las diferentes asignaturas y vemos poco probable que mida el aprendizaje significativo. De todas formas, la 
variable que hace referencia a la motivación intrínseca de los estudiantes hacia el aprendizaje (metas de aprendizaje) ha sido excluida del modelo también con signo negativo.

Lo que sí está claro es que la variable que mejor predice el rendimiento académico es el autoconcepto académico, lo cual nos sitúa ante el postulado de Marsh (1990), o más recientemente de Muijs (1997), sobre la determinación del rendimiento por el autoconcepto, defendiendo el modelo del «self enhancement» o la mejora del rendimiento a través del cambio del autoconcepto (Marsh, 1987; Shavelson y Bolus, 1982; Song y Hattie, 1984). De esta manera, si el autoconcepto del estudiante es fuerte y positivo y tiene confianza en sí mismo para emprender tareas nuevas, seguramente creerá en un éxito futuro y los fracasos que pueda experimentar no alterarán sus autopercepciones (Rogers, 1982).

No queremos finalizar sin antes comentar una de las limitaciones con que nos hemos encontrado, que ha sido la de obtener información sobre las estrategias de aprendizaje que utilizan los estudiantes a través de un cuestionario de autoinforme, de manera que los estudiantes se tienen que imaginar cualquier situación y decidir si utilizan alguna estrategia o no. Para futuras ocasiones proponemos medir la utilización de éstas mediante la realización de pruebas concretas y contextualizadas. Además, como hemos señalado anteriormente, no hemos podido recoger información relevante acerca de otros aspectos muy importantes dentro de este tema como son las estrategias de transfer, es decir, sería muy interesante y beneficioso conocer por qué los estudiantes, después de haber aprendido y dominado los contenidos, no son capaces de aplicarlos en otras situaciones diferentes y, por supuesto, qué tipo de instrumentos se pueden proporcionar para solucionar este problema.

Aunque, anteriormente, se ha mencionado ya, queremos insistir en que, a pesar de la gran importancia que tiene el contexto y todos los factores que lo componen, no ha sido tratado y esto ha provocado que los modelos de regresión, obtenidos con las diferentes variables criterio, hayan tenido una explicación tan baja de la variabilidad de las mismas. Nos hemos centrado, únicamente, en el estudiante y, además, con el sesgo que pueden producir los cuestionarios de autoinforme. Por lo tanto, en posteriores trabajos de este tipo, es de especial relevancia incluir las variables situacionales que pueden favorecer o perjudicar las demás que están influyendo en el proceso de aprendizaje. Así, hubiera sido interesante recoger algún tipo de información sobre el tipo de enseñanza utilizada por el profesor, comprobar si actúa de manera diferente según las asignaturas de que se trate, estudiar el tipo de control que ejerce en el proceso de aprendizaje de sus alumnos, de manera que nos permita comprobar si existe algún tipo de relación entre las estrategias de enseñanza utilizadas por el profesor y las de aprendizaje empleadas por los estudiantes.

En cuanto al aprendizaje autorregulado, bien es verdad que lo que es la autorregulación en sí no se puede medir directamente a través de un cuestionario puesto que la descontextualizaríamos y nos centraríamos más en los componentes que en el proceso. Por ello, una posible línea de investigación iría por ese camino, es decir, entender el proceso de autorregulación como una macroestrategia y comprobar cómo el estudiante sigue todos los pasos necesarios para regular su propio aprendizaje. Sería interesante descubrir si, en esa secuencia de pasos, ocurre algo si se salta alguno de ellos. Nos podríamos plantear las siguientes cuestiones: ¿Se puede aprender a autorregular el propio comportamiento? ¿Cómo se produce? ¿Cuál es el papel de los profesores? ¿Cómo se puede lograr en la familia? 


\section{Referencias bibliográficas}

Ames, C. (1992). Classrooms: Goals, structures, and student motivation. Journal of Educational Psychology, 84, 261-271.

Brookover, W. B. (1967). Self-concept of ability and school achievement. III Final Report on Cooperative Research Proyect, $n^{\circ}$. 2831. Michigan State University East Lansing.

Byrne, B. M. y SHavelson, R. J. (1986). On the structure of adolescent self-concept. Journal of Educational Psychology, 78, 474-481.

Cabanach, R. (1994). Modelo cognitivo-motivacional en niños con y sin «DA». Investigación original. Universidade da Coruña.

Covington, M. V. (1985). Strategic thinking and the fear of failure. En J. V. Segal, S.F. Chipman y R. Glaser (eds.): Thinking and learning skills. Vol.1. Hillsdale, NJ: Erlbaum.

Covington, M. V. y Omelich, C. L. (1984). Task-oriented versus competitive learning structures: motivational and performance consequences. Journal of Educational Psychology, 76, 1038-1050.

Dweck, C. S. (1986). Motivational processes affecting learning. American Psychologist, 41, 10401048 .

Dweck y Leggett, E. (1988). A social-cognitive approach to motivation and personality. Psychological Review, 95, 256-273.

Elig, T. y Frieze, I. H. (1979). Measuring causal attributions for succes and failure. Journal of Personality and Social Psychology, 37, 621-634.

Findley, M. J. y Cooper, H. M. (1983). Locus of control and academic achievement: A Literature Review. Journal of Personality and Social Psychology, 44, 419-427.

González, M. C. y Tourón, J. (1992). Autoconcepto y rendimiento académico. Sus implicaciones en la motivación y en la autorregulación del aprendizaje. Pamplona: EUNSA.

Hattie, J. (1992). Self-concept. Hillsdale, NJ: Lawrence Erlbaum Associates.

Hayamizu, T. y Weiner, B. (1991). A test Dweck's model of achievement goals as related to perceptions of ability. Journal of Experimental Education, 59, 226-234.

Heider, F. (1958). The psychology of interpersonal relations. New York: Wiley.

Lan, W. Y. (1996). The effects of self-monitoring on students course performance, use of learning strategies, attitude, self-judment ability, and knowledge representation. The Journal of Experimental Educational, 64(2), 151-171.

Marsh, H. W. (1984). Relations among dimensions of self-attribution, dimensions of self-concept, and academic achievements. Journal of Educational Psychology, 76, 1291-1308.

Marsh, H. W. (1987). The hierarchical structure of self-concept an application of hierarchical confirmatory factor analysis. Journal of Educational Measurement, 24, 17-39.

Marsh, H. W. (1990). The structure of academic self-concept. The Marsh/Shavelson model. Journal of Educational Psychology, 82, 623-636.

Marsh, H. W., Parker, J. y Barnes, J. (1985). Multidimensional adolescent self-concepts: Their ralationship to age, sex and academic measures. American Educational Research Journal, 22, 422444.

Marsh, H. W. y Hattie, J. (1996). Theoretical perspectives on the structure of self-concept. In B. A. Bracken (Ed.), Handbook of self-concept (pp. 38-90). New York: Witey.

Mayer, R. E. (1992). Cognition and instruction: Their historic meeting within Educational Psychology. Journal of Educational Psychology, 84 (4), 405-412. 
Muijs, R. D. (1997). Predictors of academic achievement and academic self-concept: a longitudinal perspective. British Journal of Educational Psychology, 67, 263-277.

Nicholls, J. G. (1984). Achievement motivation: Conceptions of ability, subjective experience, task choice, and performance. Psychological Review, 91, 328-346.

Nicholls, J. G. (1989). The competitive ethos and democratic education. Cambridge, M.A.: Harvard Univ. Press.

Núñez, J. C. y González-Pienda, J. A. (1994). Escala de evaluación de procesos y contextos motivacionales. Departamento de Psicología. Universidad de Oviedo.

Núñez, J. C., González-Pienda, J. A., García, M., González-Pumariega, S., Roces, C.; Álvarez, L. y González, M. C. (1998). Estrategias de aprendizaje, autoconcepto y rendimiento académico. Psicothema, 10(1), 97-109.

Pressley, M. y Ghatala, E. S. (1990). Self-regulates learning: Monitoring learning from text. Educational Psychologist, 25, 19-34.

Rogers, C. R. (1982). Psicología Social de la Enseñanza. Madrid: Visor Libros.

Rogers, C. R. (1995). Science post-16 and motivational orientation patterns. Conference paper, Annual Conference of AERA. San Francisco, C.A.

Schunk, D. H. (1991). Self-efficacy and academic motivation. Educational Psychologist, 26, 207-231.

Seifert, T. L. (1997). Academic goals and emotions: Results of a structural equation model and a cluster analysis. British Journal of educational Psychology, 67, 323-338.

Seligman, M. P. (1975). Learned helpssness: On depression, development an death. Freeman, San Francisco.

Shavelson, R. J., Hubner, J. J., Stanton, G. C. (1976). Self-Concept: Validation of Construct Interpretations. Review of educational Research, 46 (3), 407-441.

Shavelson, R. J. y Bolus, R.(1982). Self-Concept: The Interplay of Theory and Methods. Journal of Educational Psychology, 74 (1), 3-17.

Smiley, P. A. y Dweck, C. S. (1994). Individual differences in achievement goals among young children. Child Development, 65, 1723-1743.

Song, I. S. y Hattie, J. A. (1984). Home environment, self-concept, and academic achievement: A causal modeling approach. Journal of Educational Psychology, 76, 1269-1281.

Weiner, B. (1979). A theory of motivation for some classroom experiences. Journal of Educational Psychology, 71, 3-25.

Weiner, B. (1986). An attributional Theory of Motivation and Emotion. New York: Springer-Verlag.

Weinstein, C. E. (1987). LASSI (Learning and Study Strategies Inventory). Clearwater, FL: H \& H. Publishing Co. 